\title{
Microwave assisted Wolff rearrangement: A facile method for the synthesis of Fmoc- $\beta$-amino acids
}

\author{
Basanagoud S. Patil, Ganga-Ramu Vasanthakumar \& Vommina V. Suresh Babu* \\ Department of Studies in Chemistry, Central College Campus, Bangalore University, Bangalore, India \\ (*Author for correspondence, e-mail: hariccb@rediffmail.com)
}

Received 30 September 2002; Accepted 5 January 2003

Key words: $\beta$-amino acids, microwave irradiation, $N$-Fmoc- $\alpha$-aminodiazoketones, Wolff rearrangement

\begin{abstract}
Summary
The Wolff rearrangement of $\alpha$-diazoketones, derived from Fmoc- $\alpha$-amino acids, under no base conditions on exposure to microwave irradiation for 40 to $60 \mathrm{sec}$ to Fmoc- $\beta$-amino acids with retention of configuration in good yield (91-95\%) is described.
\end{abstract}

\section{Introduction}

The $\beta$-peptides, oligomers of $\beta$-amino acids, have particular appeal in understanding of protein structure and stabilization [1]. They are chemically stable, resistant to $\alpha$-peptidases [2] and mimic the activities of peptide toxins and antibiotics [3]. In view of these recent findings, there is a need to develop a facile way for the synthesis of the monomers. The Wolff rearrangement of $\alpha$-diazoketones derived from $N$ urethane protected $\alpha$-amino acids is a key step in the convenient approach to optically pure $\beta$-amino acids through an Arndt-Eistert homologation [4] because, the rearrangement proceeds with retention of configuration at the chiral centre next to the carbonyl group. It can be accomplished thermally [4], photochemically [4] or by ultrasonication [5].

The rearrangement of Boc-/Z-protected aminodiazoketones when carried out using silver oxide/ $\mathrm{Na}_{2} \mathrm{CO}_{3} / \mathrm{Na}_{2} \mathrm{~S}_{2} \mathrm{O}_{3} \cdot 5 \mathrm{H}_{2} \mathrm{O}$ by refluxing at $80^{\circ} \mathrm{C}$ for $1-3 \mathrm{~h}$ afforded the corresponding $\beta$-amino acids in about $75 \%$ yield [6]. Leggio et al. [7], synthesized Fmoc- $\beta$-amino acids in about $50-90 \%$ yield by refluxing the $\alpha$-diazoketone at $70^{\circ} \mathrm{C}$ in the presence of $\mathrm{C}_{6} \mathrm{H}_{5} \mathrm{COOAg}$ for about $1-3 \mathrm{~h}$. The ultrasound promoted rearrangement of $\alpha$-diazoketones at r.t. in presence of $\mathrm{C}_{6} \mathrm{H}_{5} \mathrm{COOAg}$ took place in about $30 \mathrm{~min}$ to give Fmoc- $\beta$-amino acids in about $70-82 \%$ yield [5]. On the other hand, the $\mathrm{Ag}^{+}$catalyzed decompos- ition of $\alpha$-diazoketones by employing about 3 equiv. of a tertiary base was also utilized [8]. Under these conditions, the reactions have to be carried out for about 3-12 h and resulted in lower yields of Fmoc- $\beta$ amino acids when compared to Boc-/Z- $\beta$-amino acids [9-11]. The present communication deals with the decomposition of $N$-Fmoc- $\alpha$-aminodiazoketones under microwave irradiation [12].

\section{Materials and methods}

Melting points were determined using capillary method and are uncorrected. IR spectra were recorded on a Nicolet model impact 400D FT-IR spectrometer ( $\mathrm{KBr}$ pellets, $3 \mathrm{~cm}^{-1}$ resolution). HPLC analysis was carried out with a Waters LC-3000 system using a C18 Bondapack $(3.9 \times 300 \mathrm{~mm}, 10 \mu)$ and chiralcel OD $(4.6 \times 250 \mathrm{~mm}, 10 \mu)$ columns with a linear gradient of water $(0.1 \%$ TFA) and acetonitrile $(0.1 \%$ TFA), with acetonitrile from 20 to $90 \%$ over $25 \mathrm{~min}$. ${ }^{1} \mathrm{H}$ NMR spectra were recorded on a Bruker AMX $400 \mathrm{MHz}$ spectrometer. Specific rotations were recorded on Rudolf Research Autopol IV automatic polarimeter. All the reactions were performed in a LG domestic microwave oven (2450 MHz) operating at $60 \%$ power. 
<smiles>[R]C(NC(F)F)C(=O)C=[N+]</smiles>

1a-k
Silver benzoate/ 1,4-dioxane, water<smiles>CC</smiles>

M.W., 40-60 sec.<smiles>[R][C@H](CC(=O)O)NC(=O)O</smiles>

$\mathbf{2 a - k}$

\begin{tabular}{|llll|}
\hline Compound & $\mathbf{R}$ & Compound & $\mathbf{R}$ \\
$\mathbf{2 a}$ & $\mathrm{H}$ & $\mathbf{2 g}$ & \\
$\mathbf{2 b}$ & $\mathrm{CH}$ & $\mathbf{2 h}$ & $\mathrm{C}_{6} \mathrm{H}_{5}$ \\
$\mathbf{2 c}$ & $\mathrm{CH}\left(\mathrm{CH}_{3}\right)_{2}$ & $\mathbf{2 i}$ & $\mathrm{CH}_{2} \mathrm{COO}^{t} \mathrm{Bu}$ \\
$\mathbf{2 d}$ & $\left.\mathrm{CH}_{2} \mathrm{CH}_{2} \mathrm{CH}_{3}\right)_{2}$ & $\mathbf{2 j}$ & $\mathrm{CH}_{2} \mathrm{COO}^{+} \mathrm{Bu}$ \\
$\mathbf{2 e}$ & $\left.\mathrm{CH}_{2} \mathrm{CH}_{3}\right) \mathrm{CH}_{2} \mathrm{CH}_{3}$ & $\mathbf{2 k}$ & $\left(\mathrm{CH}_{2}\right)_{4} \mathrm{NHBoc}$ \\
$\mathbf{2 f}$ & $\mathrm{CH}_{2} \mathrm{C}_{6} \mathrm{H}_{5}$ & & \\
\hline
\end{tabular}

Scheme 1.

Table 1. Charactaristic data for the $\beta$-amino acids prepared

\begin{tabular}{|c|c|c|c|c|c|c|}
\hline Sl. No. & $\begin{array}{l}\text { Compound } \\
2\end{array}$ & $\begin{array}{l}\text { Time } \\
(\mathrm{sec})\end{array}$ & $\begin{array}{l}\text { m.p. } \\
\left({ }^{\circ} \mathrm{C}\right)\end{array}$ & $\begin{array}{l}\text { Yield } \\
(\%)\end{array}$ & $\begin{array}{l}{[\alpha]_{D}^{25}} \\
\left(\mathrm{c}=1, \mathrm{CHCl}_{3}\right)\end{array}$ & $\begin{array}{l}{ }^{1} \mathrm{H} \text { NMR } \\
\left(\delta, \mathrm{CDCl}_{3}\right)\end{array}$ \\
\hline 1 & $\mathbf{a}$ & 50 & 148 & 91 & - & $\begin{array}{l}2.75(2 \mathrm{H}, \mathrm{t}), 3.4(2 \mathrm{H}, \mathrm{m}), 4.22(1 \mathrm{H}, \mathrm{t}), 4.6(2 \mathrm{H}, \mathrm{d}), 5.55(1 \mathrm{H}, \mathrm{br}), \\
7.25-7.85(8 \mathrm{H}, \mathrm{m})\end{array}$ \\
\hline 2 & b & 40 & 98 & 92 & -21.0 & $\begin{array}{l}1.12(3 \mathrm{H}, \mathrm{d}), 2.3(1 \mathrm{H}, \mathrm{d}), 2.45(1 \mathrm{H}, \mathrm{d}), 3.86(1 \mathrm{H}, \mathrm{m}), 4.1-4.35(3 \mathrm{H}, \\
\mathrm{m}), 5.98(1 \mathrm{H}, \mathrm{br}), 7.25-7.85(8 \mathrm{H}, \mathrm{m})\end{array}$ \\
\hline 3 & c & 40 & 152 & 95 & -36.2 & $\begin{array}{l}0.94(6 \mathrm{H}, \mathrm{d}), 1.9(1 \mathrm{H}, \mathrm{m}), 2.47(2 \mathrm{H}, \mathrm{d}), 3.85(1 \mathrm{H}, \mathrm{m}), 4.17-4.33 \\
(3 \mathrm{H}, \mathrm{m}), 6.43(1 \mathrm{H}, \mathrm{br}), 7.27-7.8(8 \mathrm{H}, \mathrm{m})\end{array}$ \\
\hline 4 & $\mathrm{~d}$ & 45 & 101 & 94 & -12.0 & $\begin{array}{l}0.8(6 \mathrm{H}, \mathrm{d}), 1.15(2 \mathrm{H}, \mathrm{m}), 1.45(1 \mathrm{H}, \mathrm{m}), 2.35(2 \mathrm{H}, \mathrm{m}), 3.8(1 \mathrm{H}, \mathrm{m}) \text {, } \\
4.25-4.4(3 \mathrm{H}, \mathrm{m}), 6.75-7.8(9 \mathrm{H}, \mathrm{m})\end{array}$ \\
\hline 5 & e & 50 & 98 & 95 & -16.8 & $\begin{array}{l}0.8(6 \mathrm{H}, \mathrm{m}), 1.35(2 \mathrm{H}, \mathrm{m}), 1.5(1 \mathrm{H}, \mathrm{m}), 2.3(2 \mathrm{H}, \mathrm{m}), 4.2-4.4(3 \mathrm{H}, \\
\mathrm{m}), 6.8-7.85(9 \mathrm{H}, \mathrm{m})\end{array}$ \\
\hline 6 & f & 40 & 112 & 95 & -26.0 & $\begin{array}{l}2.55(2 \mathrm{H}, \mathrm{m}), 2.9(2 \mathrm{H}, \mathrm{d}), 4.1-4.35(4 \mathrm{H}, \mathrm{m}), 6.52(1 \mathrm{H}, \mathrm{br}), 7.16- \\
7.85(13 \mathrm{H}, \mathrm{m})\end{array}$ \\
\hline 7 & $\mathbf{g}$ & 60 & 97 & 92 & -22.0 & $2.5(2 \mathrm{H}, \mathrm{d}), 4.2-4.35(4 \mathrm{H}, \mathrm{d}), 5.9(1 \mathrm{H}, \mathrm{br}), 7.25-7.85(13 \mathrm{H}, \mathrm{m})$ \\
\hline 8 & $\mathrm{~g}^{\mathrm{a}}$ & 60 & 98. & 91 & +21.8 & $\begin{array}{l}2.45(2 \mathrm{H}, \mathrm{d}), 4.23(2 \mathrm{H}, \mathrm{m}), 4.35(2 \mathrm{H}, \mathrm{d}), 5.85(1 \mathrm{H}, \mathrm{br}), 7.25-7.85 \\
(13 \mathrm{H}, \mathrm{m})\end{array}$ \\
\hline 9 & $\mathbf{h}$ & 50 & 82 & 92 & $+0.3^{b}$ & $\begin{array}{l}1.45(9 \mathrm{H}, \mathrm{s}), 2.65(4 \mathrm{H}, \mathrm{m}), 4.15-4.45(4 \mathrm{H}, \mathrm{m}), 6.45(1 \mathrm{H}, \mathrm{br}), 7.25- \\
7.8(8 \mathrm{H}, \mathrm{m})\end{array}$ \\
\hline 10 & i & 40 & 58 & 91 & -11.4 & $\begin{array}{l}1.45(9 \mathrm{H}, \mathrm{s}), 2.45(4 \mathrm{H}, \mathrm{m}), 3.85-4.5(4 \mathrm{H}, \mathrm{m}), 5.7(1 \mathrm{H}, \mathrm{d}), 7.25-7.9 \\
(8 \mathrm{H}, \mathrm{m})\end{array}$ \\
\hline 11 & $\mathrm{j}$ & 40 & 96 & 92 & +15.7 & $\begin{array}{l}1.2(9 \mathrm{H}, \mathrm{s}), 2.5-2.65(2 \mathrm{H}, \mathrm{d}), 3.45-3.5(2 \mathrm{H}, \mathrm{m}), 4.15(1 \mathrm{H}, \mathrm{m}), 4.2- \\
4.4(3 \mathrm{H}, \mathrm{m}), 6.35(1 \mathrm{H}, \mathrm{br}), 7.3-7.8(\mathrm{H}, \mathrm{m})\end{array}$ \\
\hline 12 & k & 40 & 98 & 93 & -8.3 & $\begin{array}{l}1.45(9 \mathrm{H}, \mathrm{s}), 2.05(8 \mathrm{H}, \mathrm{m}), 2.45(2 \mathrm{H}, \mathrm{d}), 3.8(1 \mathrm{H}, \mathrm{m}), 4.15-4.35 \\
(3 \mathrm{H}, \mathrm{m}), 5.65(1 \mathrm{H}, \mathrm{br}), 7.25-7.8(9 \mathrm{H}, \mathrm{m})\end{array}$ \\
\hline
\end{tabular}

a D-Phg.

$\mathrm{b}(\mathrm{c}=1.9, \mathrm{MeOH})$.

\section{General procedure for the synthesis of Fmoc- $\beta$-amino acids}

A mixture of Fmoc- $\alpha$-aminodiazoketone $(10 \mathrm{mmol})$, 1,4-dioxane $(7 \mathrm{ml})$, water $(3 \mathrm{ml})$ and silver benzoate
(0.15 mmol) in a beaker was exposed to microwave irradiation until the reaction was completed and filtered. Aqueous $10 \%$ sodium carbonate $(50 \mathrm{ml})$ was added to the solution, stirred for few minutes, washed with ether $(2 \times 50 \mathrm{ml})$, acidified to $\mathrm{pH} 2 \mathrm{using} 2 \mathrm{~N} \mathrm{HCl}$ 
solution and extracted with ethyl acetate $(3 \times 50 \mathrm{ml})$. The organic layer was washed with water, dried over anhydrous sodium sulphate, evaporated, precipitated and recrystalized to give the title compound.

\section{Results and discussion}

It is now found that the Wolff rearrangement of 1a-k [13-15] can be accelerated under microwave irradiation. It was carried out by exposing the mixture of 1 and $\mathrm{C}_{6} \mathrm{H}_{5} \mathrm{COO}^{-} \mathrm{Ag}^{+}$in 1,4-dioxane-water $(7: 3)$ to microwaves in an unmodified domestic microwave oven (Scheme 1). The decomposition of $\mathbf{1}$, as monitored by TLC (chloroform:methanol:acetic acid, 40:2:1) and I.R., was complete in about $40-60 \mathrm{sec}$. The extraction of the $\beta$-amino acid into an aqueous sodium carbonate solution, acidification and routine work-up lead to the isolation of $\mathbf{2 a - k}$ as crystalline solids in about 91-95\% (Table 1). All the 2a-k, prepared by this method, have been characterized by ${ }^{1} \mathrm{H}$ NMR. The reaction, as monitored by HPLC analysis of Fmoc- $\beta$-HVal-OH ( $\mathrm{R}_{t}$ for L isomer $15.42 \mathrm{~min}$ and for racemic mixture (prepared specifically for analysis) $15.36 \mathrm{~min}$ and $16.12 \mathrm{~min}$ ), is found to be completely free from racemization. Thus, a basefree $\mathrm{Ag}^{+}$catalyzed Wolff rearrangement of Fmoc- $\beta$ amino acids can be accomplished under microwave irradiation efficiently. The reaction is fast and the work-up is simple. It proceeds with good yield and without racemization. Furthermore, the decomposition of $\alpha$-aminoacyldiazoketone using 1,4-dioxanemethanol mixture resulted in the concomitant formation of the methyl ester of the corresponding $\beta$-amino acid [16].

\section{Acknowledgements}

We thank DST, Govt. of India for financial assistance. V.V.S.B. thanks DBT, Govt. of India, for a overseas associate ship award. We also thank Professors K. M. Sivanandhaih and B. S. Sheshadri for useful discussions.

\section{References}

1. (a) Cheng, R. P., Gellman, S. H. and DeGrado, W. F., Chem. Rev., 10 (2001) 3219.

(b) Gellman, S. H., Acc. Chem. Res., 31 (1998) 173.

(c) Seebach, D. and Mattews, J. L., Chem. Commun., (1997) 2015 .

(d) Liu, D. and Degrado, W. F., J. Am. Chem. Soc., 123 (2001) 7553.

2. Hintermann, T. and Seebach, D., Chimia, 50 (1997) 244.

3. Hamuro, Y., Schneider, J. P. and DeGrado, W.F., J. Am. Chem. Soc., 121 (1999) 12200, and references cited there in.

4. (a) Ye, T. and McKervey, M. A., Chem. Rev., 54 (1994) 1051 (b) Cole, D. C., Tetrahedron, 50 (1994) 9517.

(c) Wolfgang, K., Eur. J. Org. Chem., (2002) 2193, and references cited there in.

5. Muller, A., Vogt, C. and Sewald, N., Synthesis, (1998) 840.

6. Plucinska, K. and Liberek, B., Tetrahedron, 43 (1987) 3507.

7. Leggio, A., Liguori, A., Procopio, A. and Sindona, G., J. Chem. Soc., Perkin Trans. 1, (1997) 1969.

8. Seebach, D., Overhand, M., Kuhnle, F. N. M. and Martinoni, B., Helv. Chim. Acta, 79 (1996) 913.

9. Guichard, G., Able, S. and Seebach, D., Helv. Chim. Acta, 81 (1998) 187.

10. Ellmerer-M, E. P., Brossner, D., Moslouh, N. and Tako, A., Helv. Chim. Acta, 81 (1998) 59.

11. Decomposition of diazoketone using $\mathrm{CF}_{3} \mathrm{CO}_{2} \mathrm{Ag}$ (0.11 equiv.)/ $\mathrm{Et}_{3} \mathrm{~N}$ (2.9 equiv.)/MeOH under argon at r.t. for $3 \mathrm{~h}$ gave $93 \%$ of Boc- $\beta$-HVal [8]; The use of NMM (2.6 equiv.) instead of $\mathrm{Et}_{3} \mathrm{~N}$, under similar conditions, gave lower yields of Fmoc compounds compared to $\mathrm{Z}$ and Boc compounds. For e.g., Fmoc- $\beta-\mathrm{HGlu}\left(\mathrm{O}^{t} \mathrm{Bu}\right)$, after $8 \mathrm{~h}$ of reaction time, was isolated in $71 \%$ yield [9].

12. (a) Abramovitch, R. A., Org. Prep. Proc. Int., 23 (1991) 683.

(b) Caddick, S., Tetrahedron, 51 (1995) 10403.

(c) Strauss, C. R. and Trainor, R. W., Aust. J. Chem., 48 (1995) 1665.

(d) Galema, S., Chem. Soc. Rev., 26 (1997) 233.

(e) Deshayes, S., Liagre, M., Loupy, A., Luche, J.-C. and Petit, A., Tetrahedron, 55 (1999) 10851.

(f) Loupy, A., Petit, A., Hamelin, J., Texirs-Boullet, F., Jacquault, P. and Mathe, D., Synthesis, (1998) 1213.

13. Suresh Babu, V. V., Gopi, H. N. and Ananda, K., J. Pept. Res., 53 (1999) 308.

14. Suresh Babu, V. V. and Gopi, H. N., Lett. Pept. Sci., 6 (1999) 173.

15. Anand, K., Gopi, H. N. and Suresh Babu, V. V., J. Pept. Res., 99 (2000) 289

16. (a) Fmoc- $\beta$-HVal-OMe: The rearrangement of Fmoc-Val$\mathrm{CHN}_{2}(10 \mathrm{mmol})$ using $\mathrm{C}_{6} \mathrm{H}_{5} \mathrm{COO}^{-} \mathrm{Ag}^{+}(0.15 \mathrm{mmol}) / 1,4$ dioxane $(7 \mathrm{ml}) /$ methanol $(3 \mathrm{ml})$ upon microwave. Irradiation gave Fmoc- $\beta$-HVal-OMe; m.p. $74{ }^{\circ} \mathrm{C} ;[\alpha]_{D}^{25}-26(\mathrm{c}=0.65$, $\mathrm{CHCl}_{3}$ ); I.R. $\gamma_{\max } \mathrm{cm}^{1}$ (KBr disk), $3200(\mathrm{NH}), 1740$ (CO for ester), and 1696 (CO for urethane); ${ }^{1} \mathrm{H} \mathrm{NMR}\left(\delta, \mathrm{CDCl}_{3}\right)$ : $0.95(6 \mathrm{H}, \mathrm{d}), 1.85(1 \mathrm{H}, \mathrm{m}), 2.55(2 \mathrm{H}, \mathrm{m}), 3.6(3 \mathrm{H}, \mathrm{s}), 3.8(1 \mathrm{H}$, $\mathrm{m}), 4.2(1 \mathrm{H}, \mathrm{t}), 4.45(2 \mathrm{H}, \mathrm{d}), 5.2(1 \mathrm{H}, \mathrm{d})$ and $7.2-7.85(8 \mathrm{H}$, m). (b) Fmoc- $\beta$-HIle-OMe: m.p. $72{ }^{\circ} \mathrm{C} ;[\alpha]_{D}^{25}-21$ (c $=0.46$, $\mathrm{CHCl}_{3}$ ); I.R. $\gamma_{\max } \mathrm{cm}^{1}$ (KBr disk) $3340(\mathrm{NH}), 1730$ (CO for ester), 1709 (CO for urethane) ; ${ }^{1} \mathrm{H} \mathrm{NMR}\left(\delta, \mathrm{CDCl}_{3}\right): 0.8(6 \mathrm{H}$, $\mathrm{m}), 1.2-1.6(3 \mathrm{H}, \mathrm{m}), 2.5(2 \mathrm{H}, \mathrm{m}), 3.75(3 \mathrm{H}, \mathrm{s}), 3.8(1 \mathrm{H}, \mathrm{m})$, $4.2(1 \mathrm{H}, \mathrm{t}), 4.45(2 \mathrm{H}, \mathrm{d}), 5.2(1 \mathrm{H}, \mathrm{d})$ and $7.2-7.8(8 \mathrm{H}, \mathrm{m})$. 